\title{
Head-movement interface for wheelchair driving based on inertial sensors*
}

\author{
Daniel Gomes, Filipe Fernandes, Eduardo Castro, Gabriel Pires, Member, IEEE
}

\begin{abstract}
Powered wheelchairs provide the only means of mobility for many people with severe motor disabilities. For those with both lower and upper limbs impairment, available interfaces may be either impossible or very difficult to use, as well as not very efficient. In this paper we propose an egocentric interface based on inertial sensors placed on the user's head. This interface is based on head movements that provide continuous direction and speed commands to steer the wheelchair, and allows an initial null-position of the head according to the natural posture of the user. However, the development of an inertial interface for driving a wheelchair presents two main challenges, namely, 1) the simultaneous movements of the head and the wheelchair, each one with its own coordinate system, and 2) the free unrestricted movement of the head. Therefore, the two coordinate systems need to be combined and several safety features are required to only ensure admissible commands. In this paper we describe the overall implementation and preliminary experiments that show the effectiveness of the proposed solution.
\end{abstract}

\section{INTRODUCTION}

People who suffer from chronic or acute motor impairments may become unable to use both their lower and upper limbs. For them, the commercially available options to steer a wheelchair are impossible to use (e.g., hand joystick) or may be impractical and difficult to use (e.g., chin joystick, blowing tube, head-switches) [1]. In more severe cases, where motor impairment also affects head movements, several alternative interfaces have been researched, namely based on voice commands [2], ocular movements [3], tongue movements [4], facial expressions [5], electromyography [6] and electroencephalography [7]. Most of these interfaces are not commercially available and are still limited to lab experiments. They may only provide time-sparse commands, require high levels of attention, lead to fatigue or high mental workload, and are prone to errors. Thus, to safely drive a wheelchair in real-world environments, these interfaces need to be supported by an intelligent navigation system that performs or adjusts the trajectory of the wheelchair [7]. When a person with a motor disability is still able to move his/her

*This work has been financially supported by the Project B-RELIABLE: PTDC/EEI-AUT/30935/2017 and the Project VITASENIOR-MT: SAICTPOL/23659/2016 with FEDER/FNR/OE funding through programs CENTRO2020 and FCT.

Daniel Gomes is with the Polytechnic Institute of Tomar, 2300-313 Tomar, Portugal (e-mail: dpv.gomes@sapo.pt).

Filipe Fernandes is with the Polytechnic Institute of Tomar, 2300-313 Tomar, Portugal (e-mail: filipef33@gmail.com).

Eduardo Castro is with the Polytechnic Institute of Tomar, 2300-313 Tomar, Portugal (e-mail: eddycastro@live.com.pt).

Gabriel Pires is with the Polytechnic Institute of Tomar, 2300-313 Tomar, and also with the Institute of Systems and Robotics, University of Coimbra, 3030-290 Coimbra, Portugal (corresponding author e-mail: gppires@ ipt.pt). head, the most natural solution is to use the head movements as the controller. Unfortunately, head-based commercially solutions are not universally applicable, as they are based on the use of proximity or switch sensors, requiring the user to move the head in a fixed space [8] [9]. Additionally, depending on the severity of the disability, users may not be able to use such systems since their neutral (natural) head position is very specific (e.g., cerebral palsy).

In the context of this paper, we are focused on wheelchair interfaces based on head motion. Some prototypes were developed applying inclination sensors [10], infrared cameras [11], Kinect [12], stereoscopic cameras [13], and inertial measurement units (IMU) such as gyroscopes [14] or accelerometers [15] [16] [17] [18] [19]. These interfaces are mostly based on gesture-commands, generating only discrete commands, and often have problems keeping a straight-line movement. In particular, the IMU-based approaches still suffer from drift issues. In [20], a head-interface based on inertial sensors was used to control a 6-DOF manipulator. The manipulator's coordinate system is static, which greatly simplifies the complexity and challenges of this interface compared to steering a wheelchair. Yet, the proposed solution exemplifies well the high potential of inertial interfaces. Six tetraplegic participants successfully performed pick-andplace tasks, which involved moving the manipulator, controlling a gripper, and switching between modes.

Although with high flexibility and interesting features, the use of inertial interfaces for wheelchair control poses several challenges that need to be addressed to achieve an effective and reliable implementation. In this paper, we propose an interface based on inertial sensors that allows the user to control a powered wheelchair with head movements. The user can select the starting position (null/neutral) of the head, making the system adjustable to a larger number of people with abnormal posture. The head command results from the combination of the head's coordinate system and the wheelchair's coordinate system, thereby achieving an absolute orientation of the head, regardless of the movements and inclination of the wheelchair. The system applies several levels of safety and movement limits, so that all generated head-commands allow safe and reliable driving.

\section{METHODS}

\section{A. Hardware}

A picture of the wheelchair prototype and hardware components is presented in Fig. 1. The current prototype is composed of three main modules:

Head Motion Unit (HMU) wearable headset consisting of one IMU (BNO055) and one Wi-Fi module (ESP8266-Thing), both connected via an $\mathrm{I}^{2} \mathrm{C}$ bus; 


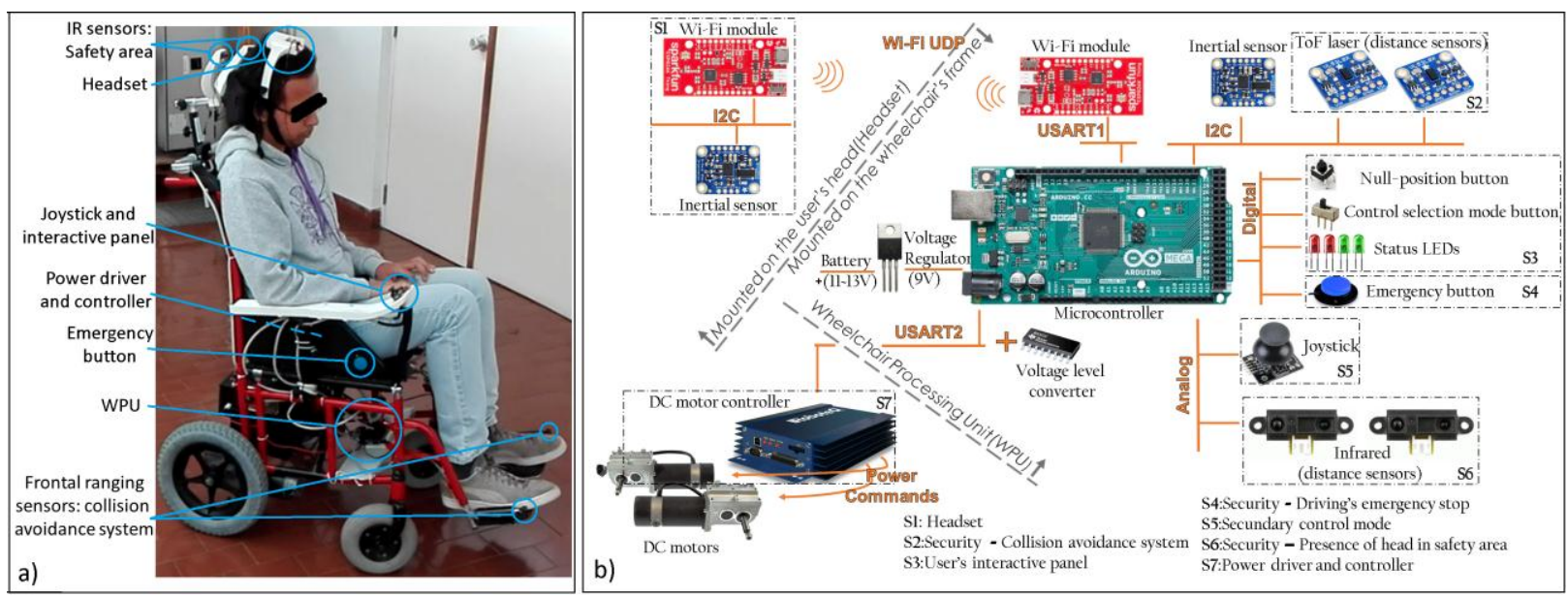

Figure 1. a) Picture of the prototype; b) Hardware components and data communication interfaces.

Wheelchair Processing Unit (WPU) and peripherals consisting of an Arduino Mega2560 microcontroller, one IMU (BNO055), one Wi-Fi module (ESP8266-Thing), and several range sensors for the safety modules. The WPU is responsible for processing the inertial data coming from the two coordinate systems, process the information related to safety restrictions and compute the final commands to drive the wheelchair. The inertial data are received from the HMU through Wi-Fi using the UDP protocol. The commands are sent via UART to a power controller (Roboteq HDC2450). There are two analog IR sensors (Sharp 2D120X) used to detect the distance of the head to the headrest, and two microlaser ToF sensors (VL53L0X) to detect frontal objects in the wheelchair's way. The Wi-Fi module is connected via UART to the Arduino, while the BNO055 and the VL53L0X sensors are connected via $\mathrm{I}^{2} \mathrm{C}$ bus. Additionally, the system integrates a biaxial joystick connected through the analog ports of the Arduino. An emergency button and several buttons and LEDs of the configuration panel are connected through digital ports;

Power system - it consists of the power driver and controller (Roboteq HDC2450) and two DC motors coupled to the rear wheels of the wheelchair.

\section{B. Wheelchair control using the user's head movements}

Two BNO055 inertial sensors provide the absolute orientation for the two coordinate systems: wheelchair and the headset (HMU). The combination of these two subsystems is called wheelchair-HMU (W-HMU). The BNO055 sensors have a fusion mode that combines the gyroscope, magnetometer and accelerometer providing an absolute threedimensional orientation in the form of either Euler angles or quaternions.

1) Two-coordinate system: The interface uses two of the three DOF that could be used for head movements (see Fig. $2 \mathrm{~b})$ ). One movement corresponds to the rotational movement of the head towards the chest (pitch - rotation around y axis), and the other is a rotation of the head towards the shoulders (roll - rotation around $\mathrm{x}$ axis). The yaw rotation was also considered, but no additional movements were required to drive the wheelchair. In all non-purely rotational movements, pitch is used to define a base speed for the wheelchair, and roll affects the angular velocity. In purely rotational movements, only roll is used, defining the speed of both motors symmetrically. Using only the coordinate system of the head to control the wheelchair would be effective only on flat surfaces. Considering that the user's body follows the orientation of the wheelchair, which in turn accompanies the surface of the floor course, the user would need to make an additional effort in the opposite direction to the floor in order to maintain pitch and roll values. The same would happen during wheelchair rotations if the yaw movement had been used. This problem is overcome by using a second coordinate system mounted on the chassis of the wheelchair, getting a two-coordinate system. Thereby, the driving command results from the difference between the head orientation and the wheelchair orientation expressed by (1) and (2):

$$
\begin{aligned}
\text { Pitch } & =\varphi_{\mathrm{c}}=\varphi_{\mathrm{h}}-\varphi_{\mathrm{w}}-\Delta \varphi_{\text {off }} \\
\text { Roll } & =\theta_{\mathrm{c}}=\theta_{\mathrm{h}}-\theta_{\mathrm{w}}-\Delta \theta_{\text {off }}
\end{aligned}
$$

where $\Delta \varphi_{\text {off }}$ and $\Delta \theta_{\text {off }}$ represent the null-position calibration offsets obtained respectively from (3) and (4).

2) Head null-position calibration: The W-HMU allows the user to define the head's initial position, which will be the null-position. This way, the interface can be customized to the posture of each user. This calibration is done whenever someone presses the calibration button for the desired nullposition. The angles of both coordinate systems in the initial position $\left(r e f_{h}, r e f_{w}\right)$ are used to compute the offset values as:

$$
\begin{aligned}
\Delta \varphi_{\text {off }} & =\varphi_{\mathrm{h}, \text { ref }}-\varphi_{\mathrm{w}, \text { ref }} \\
\Delta \theta_{\text {off }} & =\theta_{\mathrm{h}, \text { ref }}-\theta_{\mathrm{w}, \text { ref }}
\end{aligned}
$$

This calibration is exemplified in Fig. 2a). The calibration offsets are stored in the WPU, and from this point forward, all the calculations made by the WPU already take these offset values into account (see Fig. 3).

\section{Wheelchair steering commands}

The overall pipeline to obtain the final steering commands is depicted in Fig. 3. The head commands, $\varphi_{c}$ and $\theta_{c}$, ranging within the interval $\left[i_{\min }, i_{\max }\right]$, are continuously being calculated. Both angles are then scaled, through a linear function, to a new interval, $\left[o_{\min }, o_{\max }\right]$, adjusted to the admissible velocity commands, $C$, of the power driver:

$$
\mathrm{C}=\frac{\left(\mathrm{X}-\mathrm{i}_{\min }\right) \times\left(\mathrm{o}_{\max }-\mathrm{o}_{\min }\right)}{\mathrm{i}_{\max } \mathrm{i}_{\min }}+\mathrm{o}_{\text {min }}
$$




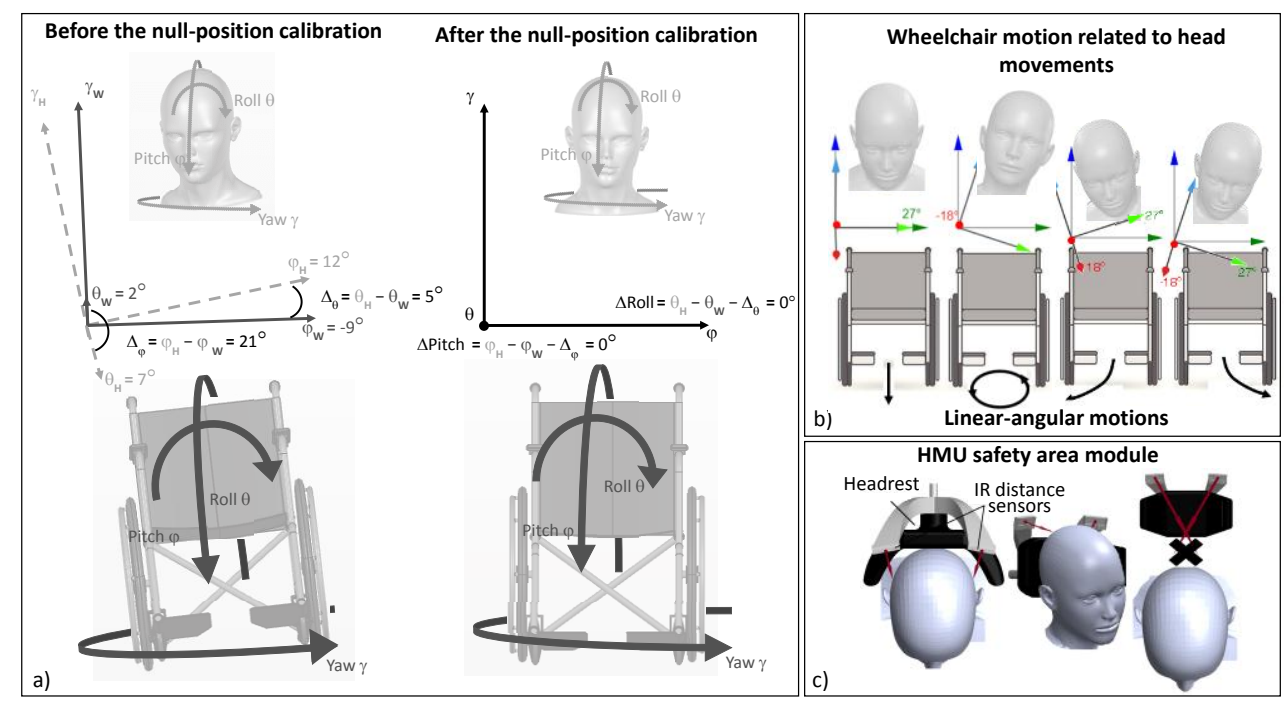

Figure 2. a) System calibration; b) Translation and rotation movements of the wheelchair; c) Head safety controlling area.

where $X$ represents $\varphi_{c}$ or $\theta_{c}$ and $i_{\min }=0^{\circ}, i_{\max }=30^{\circ}, o_{\min }=$ $150, o_{\max }=500$, are the minimum and maximum values for positive pitch and roll input, and their corresponding output velocities, and the symmetric values are applied to negative angles. The power controller applies the final commands as a percentage of the maximum motor speed.

Several safety modules were implemented to prevent inadmissible head commands, as listed below:

- If the user makes a head movement for which the angles exceed predefined limits, a null command is sent to the power controller to stop the wheelchair;

- If the head goes out of the control safety area, detected by the two ranging sensors shown in Fig. 2c), for example due to unintended actions, the wheelchair is stopped;

- If a sudden movement is made, even if the user's head is within the safety area and the angles do not exceed the predefined limits, the wheelchair's movement is smoothed due to multiple levels of acceleration and deceleration;

- If obstacles are detected by the 2 frontal sensors within a 50 $\mathrm{cm}$ range, a basic collision avoidance system limits the movements of the wheelchair. If both frontal sensors detect an obstacle within $50 \mathrm{~cm}$, only rotation commands are allowed. If only one of the sensors detects an obstacle within $50 \mathrm{~cm}$, translational commands are accepted if they are accompanied with a rotation to the opposite side.
- The wheelchair stops whenever a power failure of any of the Wi-Fi modules is detected, or a lack of communication between the two modules occurs, for at least $1.5 \mathrm{~s}$;

- Whenever the emergency button is pressed, the power controller is locked, stopping the wheelchair, until it is restarted or until it receives the command that unlocks it.

\section{EXPERIMENTAL RESULTS}

Five participants, aged from 21 to 36, all able-bodied, participated in tests using the W-HMU, and evaluated their experience. Before these experiments, the operation and functionalities of the HMU interface and wheelchair were explained and exemplified to all participants.

\section{A. Experimental procedures and evaluation criteria}

The first set of tests consisted in executing many simple movements in an open area, where participants could drive without any space constraints and learn how to control the wheelchair. These tests started with basic movements, namely forward, rotation and diagonally so the participants could adjust themselves to the wheelchair. Once participants got used to the control system, they had to climb and descend a ramp, in order to determine the effectiveness of the calibration. Then, several tests were carried out to evaluate the safety systems. Participants were asked to: 1) move against a wall or other obstacles; 2) leave the head's safety area; 3) make exaggerated head movements within the

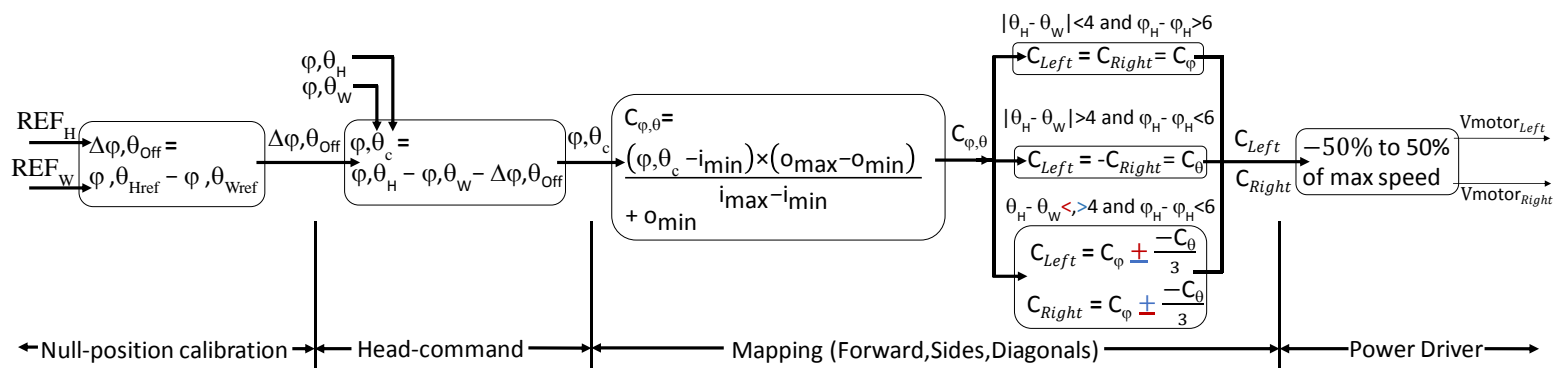

Figure 3. W-HMU command pipeline to obtain the steering commands. 
safety area; and 4) make sudden head movements. An additional test was made by an external operator which consisted in switching off the Wi-Fi modules while the wheelchair was moving. Finally, participants were asked to perform 3 times a trajectory with the shape of an eight, making a complete spin in each of the eight's extremes. The total travelled path was around 65 meters. The following evaluation criteria were used to assess the effectiveness of the HMU interface: 1) an objective evaluation, based on the time the participants took to complete the path, and number of collisions; and 2) a subjective evaluation, based on answers to a questionnaire about their experience. The questionnaire comprised the following questions specifically addressing the evaluated parameters:

Q1) How easy and intuitive were the head's movements?

Q2) How well did the wheelchair's movements correspond to the head's movements?

Q3) How fast did the wheelchair react to the head's movement?

Q4) How did you feel about the safety systems during the wheelchair's operation?

Q5) How well did the interactive panel display the information?

\section{B. Results of wheelchair driving}

Regarding the first set of random and exploratory tests, every participant felt that doing simple movements with the wheelchair was easy and intuitive, only taking a few tries to learn how to drive the wheelchair. Moving in a large open space gave the participants a sense of confidence to make them progress to more difficult movements. The results of the systematic tests are shown in Table I. The first experiment was to climb and descend a ramp. Participants reported the head movements as smooth and as easy as on a flat surface. During risky manoeuvres, participants also reported that the safety systems always worked as expected. In the path test, participants were required to make fluid and fast movements in order to complete the path in as little time as possible. The results of the questionnaire show that the head movements are intuitive and natural and that the safety modules are effective, as indicate the scores of Q1, Q2 and Q4 equal or above 4 in a scale of 1 to 5 . This agrees with participants' reported comments. The worst parameter is the reaction time, scored with 3.6, which we believe to be caused by a small latency of the overall system. Participants were able to conclude the all trajectory without collisions in $1.56 \mathrm{~min}$ on average. The overall results show that the developed control system seems to be a viable alternative to existing interfaces.

\section{CONCLUSION}

An inertial interface based on head movements was proposed to steer a wheelchair and validated successfully with healthy participants. As innovative features, the system offers the user the possibility to position his/her head in the most comfortable position, defining a customized initial neutral position, and allows to generate continuous commands of speed and direction with simple, natural and intuitive head movements. Experiments with people with motor impairment are now required for an effective system validation with the target population.
TABLE I. OBJECTIVE AND SUBJECTIVE RESUlTS

\begin{tabular}{ccccccc}
\hline Subject & Time & Q1 & Q2 & Q3 & Q4 & Q5 \\
\hline S1 & 1,45 & 4 & 4 & 3 & 5 & 5 \\
S2 & 1,33 & 4 & 4 & 4 & 5 & 5 \\
S3 & 1,54 & 5 & 4 & 3 & 5 & 5 \\
S4 & 1,35 & 4 & 5 & 5 & 4 & 3 \\
S5 & 2,13 & 3 & 3 & 3 & 5 & 5 \\
\hline Average & $\mathbf{1 , 5 6}$ & $\mathbf{4}$ & $\mathbf{4}$ & $\mathbf{3 , 6}$ & $\mathbf{4 , 8}$ & $\mathbf{4 , 6}$ \\
\hline Notes: & (Minutes) & \multicolumn{7}{c}{ (The highest score is 5 and the lowest is 1) }
\end{tabular}

\section{REFERENCES}

[1] K. Arshak, D. Buckley and K. Kaneswaran, "Review of Assistive Devices for Electric Powered Wheelchairs Navigation", ITB Journal, vol. 7, n' $1,2006$.

[2] G. Pires and U. Nunes, "A Wheelchair Steered Through Voice Commands and Assisted by a Reactive Fuzzy Logic Controller", J. of Intelligent and Rob. Syst, vol. 34, no 3, pp. 301-314, 2002.

[3] K. Arai and R. Mardiyanto,"Eyes Based Eletric Wheel Chair Control System”, Int. J. of Adv. Comp. Sc. \& App., vol. 2, n 12, 2011.

[4] X. Huo, J. Wang and M. Ghovanloo, "Wireless control of powered wheelchairs with tongue motion using tongue drive assistive technology", 30th Int. Conf. of the IEEE EMBS, 2018.

[5] J. S. Ju, Y. Shin and E. Y. Kim, "Vision based interface system for hands free control of an intelligent wheelchair", J. of Neuro Eng. and Rehab., vol. 6, n 33, 2009.

[6] G. Jang and Y. Choi, "EMG-based continuous control method for electric wheelchair”, IEEE Int. Conf on Intell. Robots and Syst, 2014.

[7] A. Lopes, J. Rodrigues, J. Perdigao, G. Pires and U. Nunes, “A New Hybrid Motion Planner: Applied in a Brain-Actuated Robotic Wheelchair", IEEE Rob. \& Autom. Mag., p. 99, 2016.

[8] ASL 105, asl, [Online]. Available: http://www.asl-inc.com/.

[9] Permobil, "Total Control Head Array System".

[10] C. Show-Hong, C. Yu-Luen, K. Te-Son, C. Chiung-Yu and H. ChaoNan, "M3S-based Electrical Wheelchair with Head-controlled Device", Proc. of the 28th IEEE EMBS Ann. Int. Conf., NY, 2006.

[11] D. J. Kupetz, S. A. Wentzell and B. F. Busha, "Head Motion Controlled Power Wheelchair”, IEEE 36th Ann. NEBEC, 2010.

[12] F. A. Kondori, S. Yousefi, L. Liu and H. Li, "Head Operated Electric Wheelchair", 2014 SE Symp. on Image Analysis and Interpretation, San Diego, CA, USA, 2014.

[13] Y. Ikushi, S. Katsuhiko and I. Takenobu, "Development of Head Gesture Interface for Electric Wheelchair", Proc. of the 1st Int. Conv. on Rehab. Eng. \& Assist. tech, Singapore, 2007.

[14] E. J. Rechy-Ramirez, H. Huosheng and K. McDonald-Maier, "Head movements based control of an intelligent wheelchair in an indoor environment”, IEEE Int. Conf. on Rob. and Biomi., China, 2012.

[15] A. Pajkanović and B. Dokić, "Wheelchair Control by Head Motion", Serbian J. of Elec. Eng., vol. 10, n 1, pp. 135-151, 2013.

[16] I. O. Qamar, B. A. Fadli, G. A. Sukkar and M. Abdalla, "Head Movement Based Control System for Quadriplegia Patients", $10^{\text {th }}$ JIEEEC, Amman, Jordan, 2017.

[17] S. Prasad, D. Sakpal and S. Rawool, "Head-Motion Controlled Wheelchair", 2017 2nd IEEE Int. Conf. RTEICT, Bangalore, 2017.

[18] Millmore, "Head Mouse - Game Controller or Disability Aid," [Online]. Available: https://www.instructables.com.

[19] C. Mandel, T. Rofer and U. Frese, “Applying a 3DOF Orientation Tracker as a Human-Robot Interface for Autonomous Wheelchairs", IEEE 10th Int. Conf. on Rehab Rob., Noordwijk, 2007.

[20] A. Jackowski, M. Gebhard and R. Thietje, "Head Motion and Head Gesture-Based Robot Control: A Usability Study”, IEEE Trans. on Neural Sys. and Rehab. Eng., 2017. 